\title{
Féeries
}

Études sur le conte merveilleux, XVII $-\mathrm{XIX}{ }^{\mathrm{e}}$ siècle

Contes et morale(s)

\section{La « morale expérimentale » du conte : l'exemple des Contes sages et fous d'Angélique Desjardins (1787)}

The "Experimental Moral" in the Fairy Tale: the Example of Contes sages et fous by Angélique Desjardins (1787)

\section{Françoise Gevrey}

\section{OpenEdition}

Journals

Édition électronique

URL : http://journals.openedition.org/feeries/1011

ISSN : 1957-7753

Éditeur

UGA Éditions/Université Grenoble Alpes

\section{Édition imprimée}

Date de publication : 15 octobre 2016

Pagination : 199-216

ISBN : 978-2-8310-335-3

ISSN : 1766-2842

Référence électronique

Françoise Gevrey, « La « morale expérimentale » du conte : l'exemple des Contes sages et fous

d'Angélique Desjardins (1787) », Féeries [En ligne], 13 | 2016, mis en ligne le 01 janvier 2017, consulté le 08 septembre 2020. URL : http://journals.openedition.org/feeries/1011 


\section{LA «MORALE EXPÉRIMENTALE» DU CONTE : L'EXEMPLE DES CONTES SAGES ET FOUS \\ D’ANGÉLIQUE DESJARDINS (1787)}

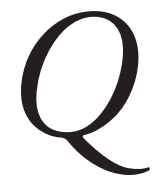

UAND ELLE FIT PUBLIER SES CONTES dans un recueil anonyme paru à Strasbourg ${ }^{\mathrm{I}}, \mathrm{M}^{\mathrm{me}}$ Desjardins choisit un titre qui, avec l'adjectif " sage $^{2}$ ", semblait préparer le lecteur à une leçon morale; mais elle remit aussi en question son sérieux en revendiquant la folie, et en jouant sur un rappel d'Érasme pour qui la Folie est une femme qui parle dans un but apologétique tout en dénonçant sa propre imposture 3 . Ce recueil, qui reprend des contes pour certains déjà publiés dans des périodiques ou pour d'autres connus dans les cercles que fréquentait la conteuse, semble répondre aux normes du temps qui imposait aux femmes une grande prudence quand elles publiaient leurs «bagatelles» sous la pression de leurs amis; le conte était alors une pratique familière, presque intime, puisqu'on sait maintenant que le mari de $\mathrm{M}^{\text {me }}$ Desjardins a fait copier une grande partie du recueil dans un beau manuscrit pour les conserver après la mort de sa femme.

S'il y a une morale dans ces contes, elle ne saurait être dissociée du contexte de sociabilité lié à la tenue d'un salon, place royale à Reims ${ }^{4}$ : mariée à un riche négociant, la conteuse jouit, au moins jusqu’à la faillite

I. Contes sages et fous, par Madame ${ }^{* * *}$, à Strasbourg, Librairie académique, 1787.

2. Sage signifie : "Qui juge, choisit, se conduit selon la raison, le bon sens", et aussi "qui se conduit selon une loi morale ou selon sa conscience. Synon. vertueux» (CNRTL).

3. Érasme affirme que "ce sont des bagatelles", qu'on y dit les choses "avec frivolité», Éloge de la folie, dans Cl. Blum, A. Godin, J.-C. Margolin et D. Ménager (éds), Érasme, Paris, Robert Laffont, coll. «Bouquins», I992, p. 8-9.

4. Voir à ce sujet A. Lilti, Le Monde des salons. Sociabilité et mondanité à Paris au XVIII siècle, Paris, Fayard, 2005, p. IIO-I20, et L. Paris, Le Théâtre à Reims, Reims, F. Michaud, I885, p. I43-152. 
de son mari qui les amena à se retirer à Cormontreuil's, d'une liberté qui permettait vers 1770 de pratiquer le théâtre avec le comte de Chambly, d'écrire des bouts-rimés, et d'avouer qu'elle aurait aimé pouvoir rivaliser avec Eisen et Boucher; sans passer pour un «bel esprit», elle participa à la rédaction de pamphlets comme à des actions de charité. Il est sûr qu'elle rencontra le "gentil Dorat», et peut-être aussi la comtesse de Genlis, passionnée par l'éducation. Le genre du conte était alors devenu un «lieu conventionnel de l'écriture au féminin ${ }^{6}$ ", la femme sensible et pédagogue pouvant le choisir pour illustrer l'importance de l'éducation face à la nature, et pour former le cœur de l'enfant à la vertu.

Sous l'influence de Rousseau, assiste-t-on alors à un retour aux objectifs moraux de Charles Perrault? $\mathrm{M}^{\mathrm{me}}$ Desjardins fait éditer ses contes au moment où se publie Le Cabinet des fées, le vaste recueil compilé par Mayer qui opère un tri en fonction de critères moraux. La conjugaison de la morale et du conte merveilleux reste cependant une entreprise difficile, dans la mesure où le merveilleux transgresse les normes, et où, dans ces contes qui ne sont pas écrits pour les enfants, l'auteur tente de concilier la morale du monde et la vertu par des allégories qui entravent l'imagination. Comme on le verra, Angélique Desjardins a évolué au fil du temps vers une morale plus affirmée qui la conduit à rivaliser explicitement avec la comtesse de Genlis.

\section{Contexte et paratexte}

À la fin du XVIII ${ }^{\mathrm{e}}$ siècle, la référence en matière de conte était moins Perrault que $\mathrm{M}^{\mathrm{me}} \mathrm{d}$ 'Aulnoy, ce qui peut avoir fait passer au second plan la stratégie de l'auteur des Contes de ma mère l'Oye, pour qui la morale signifiait la vertu et qui ajoutait la formule «avec des moralités» dans le titre de 1697. On retrouve encore ce point de vue chez l'interlocuteur que met en scène Antoine Bret dans la «Façon de préface» du ***** :

5. Il se peut que la conteuse ait écrit ses contes les plus tardifs après la ruine de son mari, comme le laisse entendre la lettre qui sert de préface à La Fée des balances, où sont évoquées «une triste saison", des "jours sombres», un "réduit» "loin de tout corps académique», Les Génies instituteurs et autres contes fin de siècle, F. Gevrey (éd.) avec le concours de S. Bénezit, Paris, Honoré Champion, coll. "Bibliothèque des Génies et des Fées», n 19 , p. 6oI. Toutes nos références aux Contes sages et fous renverront à cette édition. Cormontreuil était un lieu de villégiature des riches bourgeois de Reims, voir Il était une fois Cormontreuil, J. Vigouroux (dir.), Cormontreuil, Maury imprimeur, 1997, p. 45-48.

6. H. Krief, Vivre libre et écrire. Anthologie des romancières de la période révolutionnaire (I789I800), Oxford, Voltaire Foundation, coll. «Vif», 2005, Introduction, p. 24. 
Les contes de fées furent, je crois, inventés pour envelopper une morale simple sous les fleurs d'une imagination légère : c'était une espèce d'apologue que le merveilleux de la féerie n'empêchait pas d'être utile même aux mœurs. On les regarda d'abord comme les premières leçons de sagesse qu'on pouvait donner aux jeunes gens, et qu'ils étaient heureux qu'on eût parées de façon à les leur faire aimer .

En fait l'ambiguïté s'était vite installée ${ }^{8}$ en raison d'une ironie sousjacente au genre; pour définir des "contes galants» dans Inès de Cordoue, Catherine Bernard, influencée par Fontenelle, se déclarait prête à peindre d'abord la nature :

Que les aventures fussent toujours contre la vraisemblance, et les sentiments toujours naturels; on jugea que l'agrément de ces contes ne consistait qu'à faire voir ce qui se passe dans le cœur, et que du reste il y avait une sorte de mérite dans le merveilleux des imaginations qui n'étaient point retenues par les apparences de la vérité?.

Les adversaires du conte, comme l'abbé de Villiers, lui refusèrent bientôt toute prétention morale, ce que confirme la pratique du genre au cours du XVIII ${ }^{\mathrm{e}}$ siècle. En effet, à quelques exceptions près, fort ambiguës, comme L'Amour magot qui propose de "tirer une morale qui sait mêler l'utile à l'amusant ${ }^{10}$ " en condamnant les passions comme les moralistes, la plupart des auteurs de contes veulent seulement " amuser $^{\mathrm{II}}$ » comme Cahusac, ou satisfaire la mode comme Gautier de Montdorge dans Brochure nouvelle, en fondant leur esthétique sur le plaisir. Ce qui conduit Voisenon ou La Morlière à montrer la volupté dans toute son immoralité, et Baret à satisfaire son égocentrisme ${ }^{12}$ sans se soucier d'édifier le lecteur. Dans les années 1740 , le refus de la morale s'afficha en même temps que la parodie, et même quand se dessinait une expérience morale, comme dans La Poupée de Bibiena, elle restait très problématique ${ }^{13}$. Le rôle de Caylus, qui était pour "une morale du sentiment et de l'expérience individuelle ${ }^{14}$ »,

7. Le ***** [Bidet], histoire bavarde, Londres [Paris], I749, dans Contes, F. Gevrey (éd.), Paris, Honoré Champion, coll. "Bibliothèque des Génies et des Fées", n ${ }^{\circ} 8$, 2007, p. 853.

8. Sur l'ambiguïté, on se reportera à Raymonde Robert, «Deux exemples des relations ambiguës du conte merveilleux et de la morale : Les Aventures d'Abdalla de l'abbé Bignon, Boca de $\mathrm{M}^{\mathrm{me}}$ Le Marchand ", Féeries, n 7, «Le conte merveilleux et la fable», 20IO, p. I47-I59.

9. Inès de Cordoue, nouvelle espagnole, Paris, Martin et Georges Jouvenel, I696, p. 7-8.

Io. L'Amour magot, histoire merveilleuse, Londres, I738, dans Contes, F. Gevrey (éd.), ouvr. cité, p. 49.

II. Grigri, histoire véritable [1749], Préface de Didacque Hadeczuca, dans Contes, F. Gevrey (éd.), ouvr. cité, p. 176.

I2. Baret, Le Grelot ou les etc., etc., etc., ouvrage dédié à moi, [s. 1.], I754.

I3. Voir à ce propos la préface de Henri Lafon, dans Bibiena, La Poupée, Paris, Desjonquères, 1996.

I4. Comte de Caylus, Contes, J. Boch (éd.), Paris, Honoré Champion, coll. «Bibliothèque des Génies et des Fées", n I2, 2005, Introduction, p. 37. 
fut sans doute non négligeable quand, à la fin de sa carrière, il réaffirma son importance dans la préface de "Cadichon» et de "Jeannette»: «Je trouvais [...] une infinité de leçons de morale qui s'introduisaient dans le cœur, sous le masque de l'agrément ${ }^{15}$ ", l'allégorie permettant de "rendre la vertu aimable». Mais rien ne prouve que ce soit une référence directe pour $\mathrm{M}^{\mathrm{me}}$ Desjardins.

Les destinataires qui paraissent dans le paratexte des Contes sages et fous ne sont pas des enfants; la conteuse ne prend pas la peine de mettre en place, comme Perrault, un dispositif propice à l'exposé de leçons morales, avec un frontispice qui imposerait la présence de la nourrice, ou avec une fausse attribution à un fils auteur. Elle assume au contraire la pratique de la sociabilité mondaine : «La Bague d'oubli» est dédié à la comtesse de Preysing (qui a contribué au Recueil de ces Messieurs) et «Les Îles Flottantes" à la marquise de Saint-Germain qui doit accepter ce "conte à dormir les yeux ouverts», "cet ouvrage en l'air ${ }^{16}$. "La Fée des balances» est adressé à un ami :

Ma muse obscure et sans cabale

Fit des vers tendres et badins

Puis, pour dérider la morale

Produisit contes enfantins ${ }^{17}$.

La conteuse développe ensuite cette idée en soulignant son désir de "peindre la vertu aimable» sous le "voile de la fiction" pour revêtir des allégories qui seraient ennuyeuses ${ }^{18}$.

S'adressant à son éditeur, l'auteure ne voit dans son ouvrage que «de simples bagatelles ${ }^{19}$ ", qui correspondraient à la "frivolité de [s]on sexe»; d'où sa relative modestie : «[...] je n'ai osé produire ma raison et ma morale, que demi-cachée sous les voiles de la fiction ${ }^{20}{ }^{\prime}$; elle revendique là encore un «sens allégorique» qui permet de trouver des vérités. Elle tire ensuite la leçon, ou le "secret», de chaque conte. Elle déplore donc que le Mercure ait mutilé "La Bague d'oubli» "de manière à ne lui laisser aucun sens moral ${ }^{21}$ ", alors que le conte a pour but de donner une leçon qui consiste pour l'âme sensible à s'élever au-dessus des objets "pour trouver leur vrai

15. Ibid., p. 591-592.

16. Contes sages et fous, ouvr. cité, p. 574 pour les deux citations.

17. Ibid., p. 602.

I8. Ibid., p. 603.

19. Ibid., p. 517.

2o. Ibid.

2I. Ibid., p. 517. 
point de vue» : on pense alors à l'analyse morale de La Rochefoucauld et à «l'optique des moralistes ${ }^{22}$ ». "La Princesse Pudibonde et le Prince Parangon" se veut banalement "le parallèle du vice et de la vertu ${ }^{23}$ "; "Le Sage Alfaran" prétend "guérir l'orgueilleux», "La Fée des balances» veut peindre «la vertu aimable, le sentiment vrai, le génie tout à la fois sublime et sage ${ }^{24} »$ en la personne de l'Inconnu qui s'oppose aux autres prétendants de la princesse; quant aux "Génies instituteurs", conte écrit avant «tant de bons ouvrages sur l'éducation» dont Adèle et Théodore (I782), il devrait éduquer une élève; comme dans la forêt de Dodone où les chênes parlaient, «tout parle à l'enfance; mais tout ne devrait lui parler que pour l'instruire ${ }^{25}$ ".

Les titres, loin d'adopter la naïveté enfantine comme ceux de Caylus, font penser aux moralistes classiques : "La Bague d'oubli, ou les malheurs de la sensibilité ${ }^{26}$ ", paru dans le Journal des dames en 1778 ; "La Fée des balances, ou les apparences trompeuses» renvoie à l'anthropologie de La Rochefoucauld ${ }^{27}$ pour rappeler à l'esprit qu'on ne peut trouver la félicité dans des "surfaces trompeuses», puisque "la raison doit opérer en nous ${ }^{28}$ ", face aux dangers de l'imagination, de l'amour-propre et de la sensibilité, le malheur ramenant à une vertu qu'on veut «aimable ${ }^{29}$ ». Quant au titre des "Génies instituteurs, ou la fontaine de Sapience ${ }^{30}$ ", il renvoie à un contexte de sagesse religieuse.

Les références explicites sont limitées dans le paratexte. L'importance accordée à La Fontaine suggère un rapprochement entre le conte et l'apologue. Du reste, la conteuse se compare à «la jeune cigale / Jadis chantant

22. Voir à ce sujet L'Optique des moralistes de Montaigne à Chamfort, B. Roukhomovsky (dir.), Paris, Honoré Champion, 2005.

23. Contes sages et fous, ouvr. cité, p. 518.

24. Lettre en vers et en prose à M. Le C...M..., ibid., p. 602.

25. Ibid., p. 519.

26. On rapprochera ce titre de ceux des nouvelles de Catherine Bernard comme Les Malheurs de l'amour.

27. Prolongée par «la scène du monde» (Contes sages et fous, ouvr. cité, p. 603). On peut noter la reprise du thème dans La Princesse de Clèves et dans le titre de Courtilz de Sandras, Les Apparences trompeuses ou les Amours du duc de Nemours et de la marquise de Poyanne (1715).

28. Contes sages et fous, ouvr. cité, p. 603.

29. Ibid., p. 602.

30. La fontaine de sapience (dans des contextes religieux, notamment judéo-chrétiens) renvoie à la sagesse de celui qui possède le savoir, la science à un degré élevé, ainsi que les qualités de jugement, d'habileté, de raison, de prudence. 
soirs et matins ${ }^{31}{ }^{1}$. Elle justifie surtout l'usage de la fiction avec l'argument formulé dans «Le Pâtre et le lion ${ }^{32}$ » :

Ces idées, toutes justes qu'elles sont, feraient bâiller le lecteur, et peut-être l'auteur lui-même, si elles n'étaient revêtues du voile séduisant de la fiction ${ }^{33}$.

Angélique Desjardins montre qu'elle connaît bien Hamilton quand elle le nomme à propos du conte de son amie, "La Princesse Modeste et le Prince Fidèle», "un badinage tellement pétri de grâces et de gaieté, que l'esprit y trouve, comme dans les contes d'Hamilton, trop de plaisir pour s'occuper à y chercher de la morale ${ }^{34} »$. Enfin elle évoque "tant de bons ouvrages sur l'éducation", et plus explicitement Adèle et Théodore; à ses yeux «les immortels lauriers de $\mathrm{M}^{\mathrm{me}}$ la comtesse de Genlis ${ }^{35}$ » auraient pu remplacer tous les bouquets de son jardin de Sapience.

\section{Une anthropologie adaptée à son temps et à son milieu}

La plus grande partie des contes de $\mathrm{M}^{\mathrm{me}}$ Desjardins développe des thèmes bien connus de ceux qui ont étudié les mœurs; l'auteur les adapte à son époque en faisant place à la thématique du bonheur ou à celle de la sensibilité, et elle ne se prive pas des ressources de la satire et de l'amour.

Dans «La Bague d'oubli», sans doute le premier qu'elle ait composé, on reconnaît des lieux communs du conte : une Fée Semblant-de-rien, à qui les mères confient l'éducation de leur fille, ne sait enseigner que $l^{\prime}$ " art de dissimuler ${ }^{36}$ "; c'est elle qui va douer la petite Candine, la fille de Loyauté et de Franchise, de la plus «excessive sensibilité». La fée TouteBonne va l'élever ensuite dans son palais, et la faire voyager pour la distraire, notamment dans les Gaules sur lesquelles règne la fée Frivolide : cet épisode permet une satire de la dissipation du monde et de la jalousie des femmes. Seul le prince Rosicle est capable d'une douce sympathie, mais la méchante fée le métamorphose en épagneul; le talisman de la bague d'oubli, possédé par le génie Insouciant, doit assurer le bonheur des jeunes

31. Contes sages et fous, ouvr. cité, p. 602.

32. "La morale toujours apporte de l'ennui; / Le conte fait passer le précepte après lui. ", Fables VI, I, «Le Pâtre et le Lion».

33. Contes sages et fous, ouvr. cité, p. 603.

34. Ibid., p. 518. Bret se réfere aussi à «l'aimable comte Hamilton» qui sut «donner un peu plus de corps" aux contes, Le ${ }^{* * * * *}$, histoire bavarde, Façon de préface, dans Contes, F. Gevrey (éd.), ouvr. cité, p. 853 .

35. Contes sages et fous, ouvr. cité, p. 519.

36. Ibid., p. 523 . 
gens : «Apprenez-y à connaître les humains, et à les juger ${ }^{37}$.» À la cour d'Insouciant la jeune fille reconnaît des gens qui sont pourtant passés par les faveurs de Raisonnable :

Ma fille, lui dit la fée, on ne vient jamais ici sans passer par le pays de Raison; mais la route est trompeuse. Beaucoup de voyageurs s'égarent, et sont arrêtés en chemin par la fée Pédante qui les enferme dans un obscur palais. D'autres, déçus par le génie de l'Orgueil, se fixent auprès de lui, et deviennent esclaves des opinions humaines : espèce volatile, qui peuple seule les états de ce faux génie ${ }^{38}$.

Rosicle reprend son apparence, et la mauvaise fée montre ses vices dans un miroir de vérité, qui n’est pas sans rappeler les miroirs dont usaient les moralistes ${ }^{39}$. Les deux amoureux se marient enfin et leur cour devient le berceau des "vertus gauloises» auxquelles ils ajoutèrent «la gaieté qui fut depuis le caractère des sujets du bon roi Henri ${ }^{40}$ ». Avec un scepticisme amusé, sans condamnation rigoureuse, la conteuse fait allusion à «l'espèce du Français raisonnable», si rare selon elle. La morale vise donc ici à tempérer les excès, dans les mœurs, dans la sensibilité, dans la passion, et à convier le lecteur à adopter le bon point de vue sur le monde. Rousseau avait écrit que "c'est un fatal présent du ciel qu'une âme sensible ${ }^{4 \mathrm{I}}$ ", le conte développe plaisamment la nécessité de modérer ses émotions. Il s'agit en somme d'une morale aimable qui reste compatible avec le public du Journal des dames.

Dans «Les Îles Flottantes ou La Princesse Pudibonde et le Prince Parangon", la morale s'exprime par le truchement de la rougeur de Pudibonde qui est le thermomètre exact de ses idées, mais plus encore "celui de la sottise générale ${ }^{42}$ ». Le conte est léger, comme le montre l'anecdote du rouge dont les femmes outrent la dose pour imiter Pudibonde. Tandis que Parangon découvre Pudibonde qui doit faire son bonheur, la fée Virvouste fait enfermer la jeune fille dans une tour. Grâce aux facéties d'un nain nommé Zinzolin, la conteuse pratique alors le persiflage, par exemple avec Clignotante la fausse prude; Pudibonde apprend que «le

37. Ibid., p. 533 .

38. Ibid.

39. Voir la fable allégorique de La Fontaine, «L'Homme et son image», Pour M.L.D.D.L.R., Fables, Livre I, II, Euvres complètes, J.-P. Collinet (éd.), Paris, Gallimard, coll. «Bibliothèque de la Pléiade", I99I, t. I, p. 46.

40. Contes sages et fous, ouvr. cité, p. 534.

4I. La Nouvelle Héloïse, première partie, lettre xxvi, R. Pomeau (éd.), Paris, Classiques Garnier, 1960, p. 63.

42. Contes sages et fous, ouvr. cité, p. 578. 
tact moral qui l'éclaire» la rend "une leçon vivante» ${ }^{43}$. Les plaisanteries se font légères, par exemple quand Colifichet s'écorche le visage à cause des mouches, ce qui permet d'en railler la mode. L'auteure s'autorise un moment de scepticisme à l'égard des philosophes, quand Mirliflore prétend percer le secret de la rougeur de Pudibonde à partir de systèmes simples comme «les sens sont le mobile universel ${ }^{44}$ ». L'éloquent personnage est un piètre «instituteur» face à la jeune fille : «[...] le tact infaillible de celle-ci l'éclairant sur le vice moral de son instituteur, elle rougit outre mesure, et ne l'écouta qu'avec répugnance ${ }^{45}$. " Le livre du maître se délite en poussière sans laisser de traces, ce que la conteuse nomme un "prodige épigrammatique $^{46}$ "; et le conte de rappeler les critiques à l'égard des précieuses tandis que Mirliflore voit la rougeur comme "un épanchement de morale ${ }^{47}$ ». Quand la vertu triomphe, Zinzolin est devenu un enfant beau comme le jour; il est «l'amour pur, l'amour vrai » qui tient compte du mérite, ce qui le rend moral, son carquois est donc vide, et il a abandonné le bandeau qui le rendait aveugle. Parangon et Pudibonde "fixèrent à jamais dans leurs états les mœurs, le génie et la félicité ${ }^{48}$ ». La triade, qui vient après le rappel de la supériorité de la vertu, est chargée de donner la moralité d'un conte pourtant fort léger dans son onomastique comme dans ses péripéties; la banalité du thème moral semble avoir libéré le merveilleux et entraîné une surenchère de plaisanteries. La morale est bien ici "déridée ${ }^{49}$ ".

"Le Sage Alfaran» se fixe un autre objectif en voulant corriger un jeune prince, Zénith, dont la vanité orgueilleuse a été encouragée par sa mère Gloriane. Un vieillard, Alfaran, y incarne la sagesse, quand il offre au roi le spectacle de la création et dans une «immensité de mondes une multitude de nouveaux êtres ${ }^{5 \circ}$ ", ce qui donne envie de se dégager de la matière, et de se rapprocher des esprits célestes. Une morale chrétienne s'esquisse quand il est dit que " tout est insecte aux yeux du Créateur, et que rien n'est grand que ce qui lui ressemble ${ }^{\text {II }}$, ce qui rappelle le chapitre «Des Esprits forts» des Caractères de La Bruyère. Dès lors le prince, qui est devenu amoureux d'une fille en habit de bergère nommée Nadire et qui n'a pu la séduire

43. Ibid., p. 587, pour les deux citations.

44. Ibid., p. 596.

45. Ibid.

46. Ibid., p. 597.

47. Ibid.

48. Ibid., p. 600.

49. Ibid., p. 602, Lettre en vers et en prose à M. le C... M...

50. Ibid., p. 537. $\mathrm{M}^{\mathrm{me}}$ Desjardins a lu Fontenelle qu'elle cite par ailleurs.

5I. Ibid., p. 538 . 
sans respecter sa vertu, est embarqué dans un voyage vers un pays où il va expérimenter la morale : un mystérieux perroquet sur une barque vient lui ordonner de ramer, ce qui rappelle le perroquet qu'Antoine Hamilton met en scène dans son conte le plus célèbre, Fleur d'Épines2. Zénith arrive dans un pays inspiré des utopies du XVIII ${ }^{\mathrm{e}}$ siècle, où règne l'égalité entre les citoyens, et où la vertu jouit du bonheur. Le prince y apprend le travail et la bienfaisance, puisque «la société humaine est un échange perpétuel de talents et de services ${ }^{33} »$. Devenu secourable, Zénith entend la voix de l'humanité pour "réparer la destinée de tous les malheureux ${ }^{54}$ ». Il éprouve la bonne sensibilité, celle qui rend heureux. Avant d'arriver à l'île de la Vérité, il lui faut alors passer par l'île de la Vanité où les métamorphoses en paons, en gros singes et en mirmidons figurent le destin des sujets :

L'air de ce pays en était cause. Étant d'ailleurs extrêmement subtil, il avait la propriété de s'insinuer dans les pores et de produire un gonflement extrême dont on ne s'apercevait pas soi-même, mais que l'on voyait dans les autres. Il n'était pas possible que ces gros corps se rencontrassent sans se heurter mutuellement. Aussi les individus étaient-ils perpétuellement en guerress.

Quand le prince retrouve sa bergère, il est devenu raisonnable, conscient du néant des grandeurs; la mort de sa mère lui montre alors qu'il convient de «régler par la raison ses affections et ses désirs ${ }^{56}$ ». Les passions ne sont pas condamnées, dans la mesure où elles peuvent devenir "l'instrument du bonheur ". Le spectacle de la nature ${ }^{57}$, la force universelle de la gravitation sont une leçon : "Ainsi dans l'ordre moral les cœurs s'attirent par les qualités de l'âme et non par l'éclat factice qui environne l'individu ${ }^{58}$." Le conte se termine ainsi :

52. «Les plumes de ses ailes étaient or et azur, le reste couleur de feu et blanc: son bec et ses ongles étaient d'or, il avait la figure d'un perroquet, hors qu'il paraissait un peu plus gros.» A. Hamilton, Fleur d'Épine, dans A. Defrance et J.-Fr. Perrin (éds), Contes, Paris, Honoré Champion, coll. «Bibliothèque des Génies et des Fées", n ${ }^{\circ}$ 16, 2008, p. 462. L'oiseau guide Tarare "[...] mais que dis-je un perroquet? c'est un phénix!». Il se révèle être le frère de Tarare, et se nomme bien Phénix (p. 5IO-5II).

53. Contes sages et fous, ouvr. cité, p. 543. $\mathrm{M}^{\mathrm{me}}$ Desjardins, qui a joué dans Nanine, n'est pas loin de partager ici le point de vue qu'exprime Voltaire dans l'article «Vertu» du Dictionnaire philosophique: "Qu'est-ce que vertu? Bienfaisance envers le prochain.»

54. Ibid., p. 547.

55. Ibid., p. 549 .

56. Ibid., p. 553 .

57. On reconnaît un argument apologétique, que développe par exemple l'abbé Pluche dans son Spectacle de la nature, ou Entretiens sur les particularités de l'histoire naturelle qui ont paru les plus propres à rendre les jeunes gens curieux et à leur former l'esprit, dont l'objectif était pédagogique.

58. Contes sages et fous, ouvr. cité, p. 553. 
Les peuples, aussi heureux que leurs maîtres, éprouvèrent qu'il n'est point d'empire plus doux que celui de la vertu, et donnèrent celui d'âge d'or au règne de Zénith et de Nadire ${ }^{59}$.

Cette fois-ci, la morale prend un accent plus religieux et plus politique, en incluant la simple critique de la vanité dans un projet de bonheur des peuples.

Avec "La Fée des Balances, ou les apparences trompeuses", dont la présentation laisse à penser que le conte est surchargé de "fictions allégoriques ${ }^{60} "$, la conteuse choisit de formuler la moralité dans l'incipit, ce qui était assez fréquent dans les apologues ${ }^{6 \mathrm{~T}}$ :

Est-il rien de plus trompeur que l'apparence, ni de plus fautif que les jugements des sens? L'histoire suivante, tirée d'une ancienne chronique, servira de preuve à cette vérité $^{62}$.

La morale réside toujours pour une part dans la satire des mœurs, ainsi lorsque le conte raille la sottise du conseil de régence du royaume de la jeune Merveille, pupille de la fée des Balances: «Par malheur ces grosses têtes étaient aussi remplies de préjugés que leurs perruques étaient chargées de poudre ${ }^{63}$." Ou bien lorsqu'on explique l'usage des paniers que portent les femmes par la volonté d'imiter les balances de la fée, parodiant ainsi le conte étiologique ${ }^{64}$. En réalité cette fée pèse les individus avec des unités de mesure morales, "grains de raison, de sentiment, de franchise, de sagesse, etc. ${ }^{65}{ }^{\prime}$. L'action du conte ressemble à celle du premier chapitre de La Princesse de Babylone de Voltaire, qui était influencé par le modèle des contes de fées, et où il s'agissait de marier Formosante, la fille du roi, âgée de dix-huit ans; un oracle avait prévu une épreuve pour les prétendants : trois rois se présentent, ainsi qu'un jeune inconnu monté sur une licorne qui disparaît rapidement. L'épreuve réside chez $\mathrm{M}^{\mathrm{me}}$ Desjardins dans la découverte d'un secret : la peau présentée aux prétendants est celle d'un pou devenu éléphant. Dès lors cinq hommes, Formidaure le guer-

59. Ibid., p. 554.

6o. Ibid., p. 603.

6I. Voir par exemple chez La Fontaine "Le Loup et l'agneau» (Livre I, Io), «La Colombe et la fourmi» (Livre II, II), «L'Ivrogne et sa femme» (Livre III, 7), «Le Paysan du Danube» (Livre XI, 7).

62. Contes sages et fous, ouvr. cité, p. 605.

63. Ibid., p. 605.

64. Cette parodie est assortie d'une plaisanterie libertine : quelques incrédules cherchèrent à s'éclaircir du fait; "cette insolente curiosité fut la seule que le beau sexe ne sut jamais pardonner ", ibid., p. 606.

65. Ibid. 
rier, Astibelle roi de Loquelle, Zirphilis prince des Frivolides, Dulcidor le séducteur et l'Inconnu vont s'affronter. La morale réside d'abord dans le contraste entre les deux camps : d'une part celui des prétendants ridicules, et d'autre part celui de l'Inconnu qui lit des «livres de morale», pratique la bienfaisance et les actes vertueux. Le défilé des prétendants permet de les jauger, de les soupeser... ce que vont faire les fées, l'une avec les balances, et l'autre avec le pouvoir de faire continuer une action commencée, la durée d'une sottise éclairant sur sa nature. Comme dans une projection, des portraits mouvants et magiques feront voir chaque homme "dans toutes ses positions caractéristiques ${ }^{66} »$, ce qui en soulignera les traits moraux. Dès lors peut se développer la critique du guerrier conquérant, "ornement inutile et fatigant ${ }^{67}$ » dans la société; Zirphilis de son côté "a tout l'esprit possible, et pas le sens commun ${ }^{68}$ ", il va bientôt persifler à propos des ridicules de la cour, comme dans le conte le plus mondain. Possédé par la volupté, Dulcidor adresse ses vœux à de véritables " espèces ${ }^{69}$ » et se révèle "plus fait pour plaire que pour aimer ${ }^{70} »$; le porte-parole d'Astibel est plus grotesque encore dans son assurance d'esprit humain qui se fie aux vraisemblances et non à la vérité. L'amour volage de Dulcidor ${ }^{71}$ est prétexte à montrer le charme de l'Inconnu, mais aussi à développer des scènes ou des attitudes «comiques ${ }^{72}$ ». Un bal organisé par Zirphilis tourne au fiasco pour le prétendant : le passage de l'enivrement à la fatigue n'est pas sans rappeler le chapitre du bal dans Angola de La Morlière ${ }^{73}$.

L'objectif est de montrer «la folie des mortels qui courent après de faux plaisirs ${ }^{74}$ », et qui négligent les «jouissances bien ordonnées de cette nature qu'ils méconnaissent ${ }^{75} »$; l'amour-propre diffère de l'amour, caractérisé par sa rareté, tout comme le bonheur ${ }^{76}$ : c'est sous la forme d'une fable en prose gravée par le sage Inconnu, que sont distinguées la vraie et

66. Ibid., p. 6II.

67. Ibid., p. 612.

68. Ibid.

69. Ibid., p. 6I3. Le terme était fréquent dans les trajectoires des personnages du roman et du conte libertins.

70. Ibid., p. 620.

7I. Voir la peinture de la galanterie avec Urgandine : «[...] elle remarqua que leurs pieds se cherchaient, se trouvaient, se pressaient, et que leurs yeux se disaient quelque chose de très expressif.» Ibid., p. 622. La fée libertine va traiter Merveille de "bégueule", ibid., p. 623.

72. Ibid., p. 623.

73. Angola (1746), seconde partie, chap. Ix, «Bal du temps passé, force de l'habitude», dans Contes, F. Gevrey (éd.), ouvr. cité, p. 646-652.

74. Contes sages et fous, ouvr. cité, p. 634.

75. Ibid.

76. Voir ibid., p. 629. 
la fausse volupté. Le secret de la peau du pou a finalement été découvert en regardant le monde : «L'individu n'y reçoit-il pas toujours la mesure de la place qu'il occupe? Moins il a de consistance naturelle, plus il se gonfle, et se hausse avec facilité pour remplir l'espace donné. [...] Vous avez pu rendre l'insecte méconnaissable, vous ne pourriez faire subir au noble coursier la même métamorphose ${ }^{77}$. "L'inconnu a ainsi mis les balances dans un équilibre parfait, et se permet de peindre ensuite l'amour vrai :

Chaque jour il lui proposait des promenades champêtres, et lui expliquait les merveilles de la nature avec la netteté, la justesse et les grâces du charmant auteur de La Pluralité des mondes $^{78}$.

Cette référence à Fontenelle, qui va de pair avec la pratique des vertus sociales et à laquelle ne souscrirait pas la comtesse de Genlis, marque une proximité avec les idées des Lumières. La mère du prince, Singulière, a compris qu'il fallait rechercher "l'espèce moyenne» :

C'est dans cette classe que l'être le moins dépendant et plus éclairé conserve l'empreinte libre de la nature, le caractère personnel et les vertus sans apprêt. En un mot le peuple est brute, le grand est masque; l'homme ne se trouve qu'entre eux deux ${ }^{79}$.

Dans cette histoire de balances, tout est affaire d'équilibre entre raison et passions. Tandis que les personnages ridicules sont punis, Philomir et Merveille sont destinés au bonheur et à la vertu. Leur cour n'est pas fermée aux plaisirs "que la sagesse se permet ${ }^{80}$ ". Le paragraphe de conclusion prend un peu de distance à l'égard de la morale, puisque le manuscrit sur lequel Philomir avait écrit les rêves instructifs qu'il avait fait chez l'Enchanteur n'a pu encore se recouvrer : le rappel du principe épicurien de «la vie est un songe» permet d'enraciner l'étude des passions dans l'expérience de tous les jours.

Ces premiers contes du recueil de $\mathrm{M}^{\mathrm{me}}$ Desjardins sont donc porteurs d'une morale adaptée au monde, ouverte aux idées des Lumières tout en reprenant des lieux communs de la littérature moraliste; cette morale s'accommode de la plaisanterie et de l'amour; le merveilleux peut s'y déployer sans contrarier la morale. Ils n'ont qu'un rapport lointain avec l'enfance et veulent plutôt plaire à un public féminin qui s'étend de la noblesse à la bourgeoisie. Mais qu'en est-il lorsque la conteuse affiche des visées éducatives : le conte devient-il alors résolument «sage»?

77. Ibid., p. 636.

78. Ibid., p. 638.

79. Ibid., p. 647.

8o. Ibid., p. 654. 


\section{«Les Génies instituteurs» et $\mathrm{M}^{\mathrm{me}}$ de Genlis : une «morale expérimentale ${ }^{{ }^{8}} 》$}

Angélique Desjardins prétend avoir écrit ce conte avant la publication des ouvrages sur l'éducation de la fin du siècle, et surtout avant Adèle et Théodore (I782). Les rencontres entre les deux ouvres sont cependant troublantes, d'autant plus que le conte manifeste une indétermination générique, tout comme Adèle et Théodore. Le rapprochement du conte et du roman éducatif n'allait pourtant pas de soi, puisque $\mathrm{M}^{\mathrm{me}}$ de Genlis ne faisait pas confiance au conte merveilleux, et affichait même du mépris pour un genre qui lui paraissait ne pas éduquer les enfants :

Les contes même que $\mathrm{M}^{\text {me }}$ d'Aulnoy fit pour cet âge, ne leur conviennent pas. Il n'y en a pas un dont le sujet soit véritablement moral; l'amour en forme toujours l'intérêt; partout on y trouve une princesse aimée et persécutée, parce qu'elle est belle; un prince beau comme le jour qui meurt d'amour pour elle, et une rivale bien laide et bien méchante consumée d'envie et de jalousie ${ }^{82}$.

Elle souligne que la morale n'y est pas bonne et que les enfants sont «seulement frappés du merveilleux ${ }^{83}$ ».

Le titre de $\mathrm{M}^{\mathrm{me}}$ Desjardins renvoie cependant à un projet éducatif qu' elle entend déployer dans le conte : les génies ne sont pas là seulement pour incarner le merveilleux, mais pour remplir leur rôle d' "instituteurs». Les premières lignes mettent la vertu en avant; l'hérö̈ne naît dans un pays où cette vertu "ne tenait plus le premier rang"; son père est un "homme sage et vertueux» qui meurt à la bataille en héros ${ }^{84}$. La naissance de l'enfant et sa première éducation se font à la campagne où la veuve s'est réfugiée dans une solitude qui plaît aux "âmes sensibles ${ }^{85}$ ", et où une mystérieuse bonne femme, appelée madame Bobinette ${ }^{86}$, en réalité la fée Lucide, la réconforte, avant de "présider à l'éducation de l'enfant» nommée Pouponne.

8I. Adèle et Théodore, ou lettres sur l'éducation contenant tous les principes relatifs aux trois différents plans d'éducation des princes et des jeunes personnes de l'un et l'autre sexe, I. Brouard-Arends (éd.), Rennes, Presses universitaires de Rennes, 2006, t. I, lettre XLIX, p. 213. On sait que Sade choisira par défi, en 1795 , Les Instituteurs immoraux comme sous-titre de La Philosophie dans le boudoir.

82. Adèle et Théodore, ouvr. cité, t. I, lettre XIV, p. 94.

83. Ibid. C'est pourquoi $\mathrm{M}^{\mathrm{me}}$ de Genlis a écrit Les Veillées du château (I784) où elle reprend le cadre choisi par les conteuses (Murat, Lussan, etc.) pour donner aux enfants de plus de dix ans une "morale mise en action» (Préface).

84. Contes sages et fous, ouvr. cité, p. 655.

85. Ibid.

86. On peut supposer un jeu de référence au «Petit Chaperon rouge» de Perrault, avec aussi une allusion au rouet des nourrices. L'onomastique de ce conte appartient davantage au domaine enfantin. 
Elle prend soin de la petite fille comme le préconise Rousseau, "point de langes ni de maillots ${ }^{87}$ ", et anticipe presque sur ce que vivront Paul et Virginie $^{88}$ : pendant cinq ans l'enfant se développe ainsi, et la conteuse note, avec quelque ironie, que «plusieurs traités d'éducation furent faits à l'occasion de la sienne ${ }^{89}{ }$. Cette première étape correspond assez bien à celle que ménage $\mathrm{M}^{\mathrm{me}}$ de Genlis dans son roman pédagogique : $\mathrm{M}^{\mathrm{me}} \mathrm{d}^{\prime} \mathrm{Al}-$ mane va éduquer ses enfants dans un château à la campagne. Angélique Desjardins limite le merveilleux à des effets de lumière jusqu'à l'enlèvement de l'enfant dans un chariot de jonc tiré par de gros oiseaux qui est un souvenir du char des fées, et à sa mise au service d'une "providence" que la fée Lucide évoque, la vertu étant finalement pour la jeune fille de "se suffire à elle-même ${ }^{90}$ ".

Un autre point commun réside dans l'utilisation du décor du château pour instruire l'enfant : pour Adèle et Théodore ce sont des tapisseries et leurs devises disposées dans une galerie ${ }^{91}$, l'aménagement des appartements devant servir de leçons; pour Pouponne ce sont d'abord les bosquets des jardins du palais qui dispensent des fables et une couronne de roses ${ }^{92}$. Quinze mois plus tard, selon un plan établi, elle entre dans le château où les tableaux eux aussi peignent les fables: "Elle en reconnaît les acteurs, en saisit la morale ${ }^{93}$ » : elle est au pays de Sapience, en présence d'une fée qui lui désigne les «génies humains» qui ont donné des leçons utiles. Dès lors va se dérouler un parcours éducatif qui conduit l'enfant de bosquet en bosquet. La jeune fille commence par cueillir les Fictions morales en passant sur Télémaque une année entière ${ }^{94}$. Vient ensuite l'apprentissage

87. Contes sages et fous, ouvr. cité, p. 656. Sur le lien de $\mathrm{M}^{\mathrm{me}}$ de Genlis avec Rousseau, voir Christophe Martin, "Sur l'éducation négative chez $\mathrm{M}^{\mathrm{me}}$ de Genlis, Adèle et Théodore, Zélie ou l'Ingénue", dans Fr. Bessire et M. Reid (dir.), Madame de Genlis. Littérature et éducation, MontSaint-Aignan, Presses des universités de Rouen et du Havre, 2008, p. 5I-65. La différence avec Rousseau tient au fait que $\mathrm{M}^{\mathrm{me}}$ de Genlis veut adapter l'enfant au monde et enracine clairement la morale dans une tradition chrétienne.

88. Le roman de Bernardin de Saint-Pierre est publié en 1788.

89. Contes sages et fous, ouvr. cité, p. 657.

90. Ibid., p. 658. On peut penser à Zilia, l'héroïne de $\mathrm{M}^{\mathrm{me}}$ de Graffigny (Lettres d'une Péruvienne, 1747).

9I. Adèle et Théodore, ouvr. cité, t. I, lettre IX, p. 77.

92. Cependant elle ne peut se mirer dans le bassin du bosquet, ce qui exprime un refus de la coquetterie, comme chez $\mathrm{M}^{\mathrm{me}}$ de Genlis. Un rosier parle, comme dans «Le Prince rosier» de Catherine Bernard (dans Inès de Cordoue), mais ce n'est pas un amoureux.

93. Contes sages et fous, ouvr. cité, p. 659.

94. Fénelon est une des références de $\mathrm{M}^{\mathrm{me}}$ de Genlis, notamment pour son traité De l'éducation des filles (t. II, p. 320). 
de l'Histoire : comme $\mathrm{M}^{\mathrm{me}}$ de Genlis95, on en souligne les dangers; les ronces, les vices et les malheurs de l'humanité «affligeaient son âme». Cette découverte est complétée par celle de l'épopée, de la guerre de Troie à $L a$ Henriade ${ }^{96}$. La jeune fille peut aussi découvrir la poésie amoureuse ou tragique, avec l'opéra et le théâtre privé des acteurs qui interprètent la tragédie (Mme Desjardins avait une passion pour le théâtre auquel elle fait place dans son programme d'éducation).

Un génie vient commander cependant à Pouponne de quitter les fictions pour chercher la sagesse : Lucide confirme le danger des passions, même si elles ont éveillé ses facultés, la sagesse étant vue comme un «équilibre» entre les puissances. D'où une nouvelle étape traversant le canton des moralistes qui inclut les philosophes: il s'agit d'un bois immense avec mille routes perdues, et donc un labyrinthe aussi trompeur qu'impraticable. La sagesse y revêt ses favoris d'un éclat qui «blesse les yeux des mortels habitués aux ténèbres de l'erreur et du vice ${ }^{97}$ ». La fée invite Pouponne à converser avec tous, mais surtout avec ceux qui guident vers la source de sapience. Tandis que des perroquets sur des branches sèches incarnent les philosophes, trois années se passent avec les «vrais instituteurs» qui développent «la nature des esprits et des corps, le monde physique et le monde moral, les charmes de la vertu, les principes inébranlables du bien, l'amour de l'humanitée ${ }^{8}{ }^{\prime}$. Et contrairement aux usages du conte, c'est la jeune fille qui va délivrer un prince, pour faire un acte de bienfaisance. Celui-ci apparaît sous la forme d'un phénix dans une tendre posture avant de reprendre son apparence humaine ${ }^{99}$. Lucide déclare alors que la sagesse n'interdit pas l'amour : «[...] elle se borne à le diriger ${ }^{100}$ »; il doit être "le plaisir de votre âme et le délassement de vos travaux ${ }^{\mathrm{IOI}} »$. Parce qu'ils ont bu l'eau de sapience, Pouponne et le prince peuvent découvrir les philosophes : «Beaucoup [...] étaient

95. Adèle et Théodore, ouvr. cité, t. I, lettre XLI, p. 193-I94, où sont développés les principes des Annales de la vertu, "ne louer que ce qui mérite d'être loué», "[...] les enfants se laissent trop facilement éblouir par tout ce qui a quelque air de grandeur, et l'injustice ne peut leur paraître odieuse quand il en résulte une action brillante, et quand elle est couronnée de succès. »

96. Mme Desjardins revient sur le mythe du bon roi Henri qui figure dans les tapisseries de $\mathbf{M}^{\mathrm{me}}$ d'Almane : "Henri IV en paraît plus grand, quand il est à côté de Sully; car le mérite d'avoir su choisir un tel ministre suffirait seul pour immortaliser un prince." (Adèle et Théodore, ouvr. cité, t. I, lettre IX, p. 77).

97. Contes sages et fous, ouvr. cité, p. 663.

98. Ibid., p. 664.

99. Il est le petit-fils du roi des Philogandes (ami de la lumière et de la joie). Lucide l'a doué du goût naturel pour la vertu. Désordonnée, la fée rivale l'avait métamorphosé. La fille qui doit le sauver est passée par le pays des fictions et des systèmes.

Ioo. Contes sages et fous, ouvr. cité, p. 666.

IoI. Ibid. 
représentés sous la forme d'enfants, qui, soufflant dans des boules de savon, les enflaient, et prenaient pour rayon de lumière les légères couleurs dont elles se revêtaient ${ }^{\mathrm{IO}}$ ", ce qui n'est pas sans rappeler au lecteur le rêve de Mangogul dans Les Bijoux indiscrets de Diderot (I748) : "Que veut dire ce souffleur avec ses bulles ${ }^{103}$ ? " Les fictions romanesques, que $\mathrm{M}^{\mathrm{me}}$ de Genlis déconseille, même quand il s'agit de La Princesse de Clèves ${ }^{104}$, montrent à Pouponne «les écarts de l'esprit et du cœur ${ }^{105}$ » : "L'œil de la sagesse admire l'art, et pleure sur l'abus ${ }^{106}$.» Si le temple indique que la piété va de pair avec "les traits les plus sublimes de la vertu», et si le pays des sciences peut être parcouru sans guide, en revanche le canton des politiques est recouvert d'un brouillard épais : un télescope enchanté en fait pénétrer les détours. Comme chez $\mathrm{M}^{\mathrm{me}}$ de Genlis, la formation se distingue ensuite selon les sexes : le garçon restera dans cet observatoire pendant six mois, tandis que la fille ira apprendre les vertus sociales avec sa mère.

Il convient enfin de découvrir le pavillon des arts. Le chant et la musique charment, mais Pouponne ne les pratique pas, à la différence de ce qui se fait dans l'éducation mondaine du temps; les génies sont plutôt source de conversation, et celui de la peinture a fait des portraits pour que les jeunes gens les échangent, ce qui favorise l'amour sans les engager dans des stratagèmes que montraient les romans, tel le déguisement en peintre dans l'Histoire d'Hypolite, comte de Duglas de $\mathrm{M}^{\mathrm{me}} \mathrm{d}$ 'Aulnoy (I690).

Le retour chez la mère permet de rattraper les conventions du conte et le merveilleux. Le bonheur de la jeune fille est moins pur que celui de sa

\footnotetext{
I02. Ibid.

I03. Les Bijoux indiscrets, chap. XxxiI, dans M. Delon (dir.), Contes et romans, Paris, Gallimard, coll. "Bibliothèque de la Pléiade», 2004, p. I09. L'image de la bulle de savon serait empruntée au cinquième des Songes philosophiques du marquis d'Argens (1746) qui se moque ainsi des «systèmes». Le mot de «système» est aussi présent dans le conte de $\mathrm{M}^{\mathrm{me}}$ Desjardins. $\mathrm{M}^{\mathrm{me}}$ de Genlis est pour sa part complètement opposée aux philosophes : voir Fr. Bessire, " $\mathrm{M}^{\mathrm{me}}$ de Genlis ou l'"ennemie de la philosophie moderne" ", dans Madame de Genlis. Littérature et éducation, ouvr. cité, p. 279-289.

I04. Adèle et Théodore, ouvr. cité, t. I, lettre XL, p. I90 : «Dans les nouveaux principes d'éducation, une mère croit faire des merveilles en permettant à sa fille de lire ce qu'on appelle des romans moraux, comme, par exemple, La Princesse de Clèves, où l'on trouve, dit-on, de si beaux exemples de vertu, où l'héroïne résiste avec tant de force et de courage à la plus violente passion. En voyant l'excès du sentiment qui la domine, et les combats affreux que le devoir excite en elle, si l'on peut croire que c'est là une peinture fidèle du cœur, il faut croire aussi que l'amour est absolument indépendant de notre volonté, qu'il est inutile de s'opposer à ses progrès, et qu'alors la vertu n'est qu'un tourment de plus. Voilà un but moral bien satisfaisant."
}

I05. Contes sages et fous, ouvr. cité, p. 666.

I06. Ibid., p. 667. 
mère, un «bonheur plus facile à imaginer qu'à décrire ${ }^{107}$ ». Une intrigue amoureuse vient pimenter l'approche du dénouement, avec la rivalité d'un prince et de son ministre. La jeune fille sage est traitée de "précieuse»: "Pouponne le sut, et apprit là que la sagesse même est obligée de se revêtir des grelots de la folie pour se communiquer aux mortels ${ }^{108} "$; la réflexion renvoie à l'expérience de la conteuse et à son titre. Il reste à recueillir les feuilles de palmier que Lucide avait envoyées à la veuve : ces mémoires en train de s'écrire évoquent la fin du roman de $\mathrm{M}^{\mathrm{me}}$ de Genlis où $\mathrm{M}^{\mathrm{me}} \mathrm{d}$ 'Almane offre ses Lettres sur l'éducation: «Vous y trouverez une peinture fidèle et des mœurs et du monde ${ }^{109}$." Après l'enlèvement dont Phénix la délivre, le ministre Sans-Frein est "changé en un loup énorme, mais dépouillé des dents terribles qui font la force de cet animal», incarnant alors «la méchanceté sans pouvoir ${ }^{\mathrm{IIO}}$ »; on peut y voir un autre détournement du conte de Perrault. Comme présent de noces, Phénix et Pouponne reçoivent de la fée un palais semblable à celui de Sapience, avec une bibliothèque qui rappelle le projet pédagogique : "Ce lieu devint le lycée du pays, et fit toujours les délices du roi et de la reine ${ }^{\mathrm{III}}$.»

Si $\mathrm{M}^{\mathrm{me}}$ de Genlis a composé un roman d'un genre particulier, il en va de même pour $\mathrm{M}^{\mathrm{me}}$ Desjardins dont le conte relègue ici le merveilleux dans les marges d'un voyage éducatif où l'on énumère les divers «instituteurs». Même travestis, les écrivains et les arts bien réels se reconnaissent dans un programme chargé. Dans les deux cas, il s'agit de préparer une jeune fille au mariage, mais Angélique Desjardins fait beaucoup plus de concessions au monde et à l'amour.

Il apparaît que le recueil des Contes sages et fous, constitué avec quelque distance par rapport à l'époque de rédaction de certains textes, porte un titre qui justifie bien son rapport à la morale. Il se situe en effet à la charnière de deux époques, qui peuvent correspondre aussi à deux étapes de la vie de l'auteur : celle où elle assumait la liberté du merveilleux en cherchant, par une aimable satire, à plaire à un public mondain, et celle où elle voulut mettre la morale au premier plan dans des allégories qui devaient clairement éduquer. Cette concurrence avec le roman éducatif tente de concilier un projet de recherche de la vertu et le merveilleux, mais le choix de l'allégorie entraîne une subordination de la fiction à la morale, et une

I07. Ibid., p. 669. La conteuse est bien consciente d'aborder là un thème qui caractérise son siècle.

108. Ibid.

I09. Adèle et Théodore, ouvr. cité, t. III, lettre XLIX, p. 626.

IIo. Contes sages et fous, ouvr. cité, p. 670.

III. Ibid., p. 67I. 
rationalisation du monde des fées. L'expérience de $\mathrm{M}^{\mathrm{me}}$ Desjardins ne va pas sans tensions : elle reste sensible à l'art d'Hamilton, à la pratique du conte en société, à la satire des mœurs, et même à l'ironie. On perçoit également sa sympathie pour Fontenelle, son intérêt pour Voltaire et pour une pensée proche de celle des philosophes des Lumières : ses contes ne sont pas incompatibles avec la Théorie des sentiments agréables de Louis Levesque de Pouilly ${ }^{\mathrm{II}}$, qui était Rémois lui aussi. Quand vint la mode des ouvrages d'éducation, Angélique Desjardins voulut alors mettre en œuvre dans le conte sa propre "morale expérimentale», en rivalisant avec $\mathrm{M}^{\mathrm{me}}$ de Genlis pour faire évoluer son élève dans un monde où celle-ci devait assimiler une foule de connaissances. Même si une fée préside à cette éducation et si les «instituteurs» sont des génies, on s'interroge alors sur l'ambiguïté générique ainsi créée. En tout état de cause, la voie est ouverte pour d'autres conteuses comme $\mathrm{M}^{\mathrm{me}}$ Mérard de Saint-Just qui, dans Le Prince de l'Étoile ou la destinée merveilleuse ${ }^{\mathrm{II}}$, cette fois-ci «dédié aux enfants nés et à naître», vont pouvoir délivrer leur morale en présence de petits auditeurs.

II2. Théorie des sentiments agréables [1736], Paris, David le jeune, I749; voir en particulier les chapitres XIII «Du plaisir attaché à l'accomplissement de nos devoirs envers les autres hommes» et XIV "Du bonheur attaché à la vertu».

II3. Le Prince de l'étoile, ou la destinée merveilleuse, [s. d.; autour de I8Io], dans Les Génies instituteurs et autres contes fin de siècle, ouvr. cité, p. 959-980. 